\title{
Indicadores de gobernanza territorial de los objetivos del Plan Nacional del Buen Vivir (PNBV 2013-2017), Ecuador ${ }^{1}$
}

\author{
Territorial governance Indicators for National Plan for Good Living objectives \\ (PNBV 2013-2017), Ecuador
}

Recibido: 25 de octubre de 2016 - Revisado: 11 de octubre de 2017 - Aceptado: 17 de marzo de 2018.

\section{Olga Bravo ${ }^{2}$}

\section{Resumen}

El propósito de este artículo es analizar las variables y sus indicadores de gobernanza territorial contenidas en el Informe de la Secretaría Nacional de Planificación y Desarrollo: "Objetivos e indicadores de apoyo al Plan Nacional del Buen Vivir 2013-2017". Utilizando la taxonomía de Whittingham, se encuentran 37 indicadores de gobernanza territorial (25\%), distribuidos en: eficiencia $65 \%$, equidad $13,5 \%$, participación $10,8 \%$, rendición de cuentas $8 \%$ y, desarrollo del recurso humano $2,7 \%$; existiendo omisión de importantes indicadores en todas estas variables, carencias en la capacitación del servidor público, subestimación de procesos de rendición de cuentas y de representación de grupos de interés, así como desatención de los mecanismos institucionales de participación ciudadana, revelando un modelo de gobernanza centrado en el Estado. A nivel regional, hubo mejorías en la mayoría de los indicadores, pero estancamiento en el ámbito de la educación superior, comprometiendo a futuro los procesos de generación y transferencia de innovaciones, claves para el desarrollo.

\section{Palabras clave}

Ecuador, Buen Vivir, gobernanza territorial, indicadores

\begin{abstract}
The purpose of this article is to analyze the variables and their indicators of territorial governance contained on National Secretariat of Planning and Development Report: "Objectives and indicators of support for the National Plan for Good Living 20132017 '. Using Whittingham's taxonomy, there are 37 indicators of territorial governance $(25 \%)$, distributed in: efficiency $65 \%$, equity $13.5 \%$, participation $10.8 \%$, accountability $8 \%$ and, human resource development $2.7 \%$; there is omission of important indicators in all these variables, lack of training for the public servant, underestimation of processes of accountability and representation of interest groups, as well as neglect of the institutional mechanisms of citizen participation, revealing a focused governance model in the state. At the regional level, there were improvements in most of the indicators, but stagnation in the field of higher education, committing future processes of generation and transfer of innovations, which are keys for development.
\end{abstract}

\section{Keywords}

Ecuador, Good Living, territorial governance, indicators.

\footnotetext{
${ }^{1}$ Investigación realizada en el marco del proyecto: "Desequilibrios regionales ante las políticas de redistribución de la riqueza en el Ecuador, basadas en el sumak kawsay", financiado por la Universidad Tecnológica Empresarial de Guayaquil, Ecuador.

${ }^{2}$ Doctora en Planificación y Gestión de Desarrollo Regional, Universidad de Zulia. Magíster de Microbiología de la misma Universidad y Licenciada en Biología de la Universidad Central de Venezuela. Directora de Investigación y docente de la Universidad Tecnológica de Guayaquil, Ecuador. Correo electrónico: obravo@uteg.edu.ec

(id https://orcid.org/0000-0003-05583228

Para citar este artículo use: Bravo, O. (2018). Indicadores de gobernanza territorial de los objetivos del Plan $\mathrm{Na}-$ cional del Buen Vivir (PNBV 20132017), Ecuador. Civilizar, 18(35), 25-38. doi:10.22518/usergioa/jour/ $\operatorname{ccsh} / 2018.2 / \mathrm{a} 03$
} 


\section{Introducción}

En un análisis de las tendencias históricas que sustentan al desarrollo local en Ecuador, Valarezo (2004) destaca varias etapas: a) siglo XIX, el poder central avanza sobre la sociedad para crear el Estado nacional; b) principios del siglo XX, las regiones abandonan pretensiones federalistas para integrarse al proyecto nacional; c) décadas 1960-1970, con la modernización y el boom petrolero, las localidades y regiones son completamente subsumidas, perdiendo la mayor parte de sus funciones y atribuciones; $y$, d) década 1990, ocurre un resurgimiento de lo local en el contexto del agotamiento del modelo centralista, la caída de los precios petroleros y otras fuerzas políticas y económicas.

Verdesoto y Ardaya (2011) destacan la modalidad rentista y las formas de clientelismo territorial asociadas a la era petrolera, pero también el papel que desempeñaron después las redes de provincias y ciudades durante la crisis general de los años noventa, impidiendo que tuviera consecuencias catastróficas; llegando a los tiempos actuales en los que las demandas de descentralización, la nueva Constitución de 2008 y las normativas territoriales correspondientes, constituyen los parámetros del desarrollo regional y local.

En tal sentido, la Constitución nacional se fundamenta en una relación sociedad-Estado-mercado bajo un régimen de desarrollo considerado como un conjunto organizado, sostenible y dinámico de los sistemas económicos, políticos, socio-culturales y ambientales, en función de garantizar la realización del buen vivir. La participación social es invocada en diferentes títulos y capítulos del texto, para garantizar el acceso de los ciudadanos en los distintos niveles de gobierno, en la formulación, ejecución, evaluación y control de las políticas y, en la planificación para el desarrollo. El PNBV 2013-2017 trata de articular la política pública nacional con las condiciones de los diversos territorios, de modo que sus lineamientos se con- cretan con los procesos de desconcentración y descentralización del Estado, en la expresión de nueve Agendas Zonales que acoplan la acción pública desconcentrada del Estado Central con el nivel descentralizado. Estas zonas están conformadas por provincias, de acuerdo a una proximidad geográfica, cultural y económica.

No obstante, Torres (2016, p.116) señala retrocesos en el ámbito del desarrollo local luego de más de ocho años de la Constitución y seis de las leyes, “... un preocupante desencuentro entre la implementación desconcentrada de las políticas del gobierno central y la descentralización de los gobiernos municipales, una tenue asunción de competencias territoriales evidencia la crónica debilidad institucional municipal que limita sus roles...”.

En ese contexto referencial, se aborda específicamente la interrogante: ¿Cuáles son las variables e indicadores de gobernanza territorial que maneja la Secretaría Nacional de Planificación y Desarrollo (SENPLAdes), como expresión de las políticas gubernamentales desde distintos niveles institucionales y a diferentes escalas espaciales? A tal fin, luego de esta breve introducción, se presentan las perspectivas teóricas de la gobernanza, la metodología utilizada para evaluar los indicadores, la discusión acerca de su evolución en las diferentes zonas de planificación y las consideraciones finales.

\section{Fundamentos teóricos}

Existe un espectro de significados de gobernanza que van desde una definición centrada en el Estado (Peters y Pierre, 2005; Peters, 2011) hasta una perspectiva policéntrica, con énfasis en otros actores del sistema (O'Toole, 2005; Rhodes, 2005; Revesz, 2006; Jorquera, 2011; Canto, 2012; Vigil y Ockstat, 2015). No obstante, el punto es que este concepto es esencial para comprender cómo funciona el sector público actual en un contexto de actores internacionales, nacionales y locales, que supone, según Cerrillo (2005), una modificación de las 
relaciones Estado-sociedad para hacer frente a los diversos intereses, recursos y visiones de los diferentes actores. En palabras de Guttman (2004), la gobernanza viene a ser un medio político para ajustar el equilibrio de poder en la sociedad.

Desde la perspectiva centrada en el Estado, Peters (2011, p.106) utiliza el término gobernanza "...como una meta que direcciona la actividad, que requiere instrumentos para identificar lo que la sociedad desea que se haga y, luego, examinar los medios para alcanzar las metas colectivas". Siendo el foco la prosecución de metas colectivas, requiere de una dirección que sea aceptada por la mayoría, de mecanismos para resolver los conflictos de intereses y asegurar la responsabilidad - rendición de cuentas- de los actores involucrados, por lo que el Estado es la única institución capaz de realizar esta tarea de forma efectiva.

Desde una perspectiva policéntrica, se argumenta una nueva forma de pensar acerca de los límites y capacidades de las estructuras estatales y, en particular, sobre las relaciones Estado-sociedad que permiten la emergencia de procesos de desarrollo local, mediante redes de actores o de grupos de interés presentes en la sociedad civil. Klijn (1998:34), plantea que estas redes “... son patrones más o menos estables de relaciones sociales entre actores interdependientes, que toman forma en torno a problemas y/o programas políticos". Similarmente, Natera (2004) considera que la gobernanza se refiere a los patrones emergentes de un sistema sociopolítico en virtud de la interacción de los actores.

Esta perspectiva ha generado diversos conceptos de gobernanza, todos relacionados: territorial, local, multinivel, policéntrica. Para la Comisión Europea (2008), la gobernanza territorial alude a una responsabilidad política compartida y colaboración mutua entre las políticas económicas, sectoriales y de desarrollo; mientras que la gobernanza local, trata del poder, de la autoridad, y de la gestión en el ámbito regional, municipal o comunal. Otros autores inscriben la discusión en el tema de la descentralización, vista como un cambio de la estructura societal, en tanto involucra a todo el tejido político y social de una nación (Boisier, 2004; 2011); desde la cual las políticas territoriales requieren considerar la gobernanza multinivel (Galicchio, 2010), la reforma de las instituciones, el fortalecimiento de las redes de actores locales y el cambio de pautas culturales (Arocena, 2002; 2011).

La gobernanza policéntrica ha sido un eje de referencia para Ostrom $(2005 ; 2009 ; 2010)$, en el análisis inductivo de problemas de acción colectiva, concretamente, la diversidad de acuerdos institucionales requeridos para abordar problemas a múltiples escalas; evidenciando cómo las instituciones - entendidas como las reglas de juego (reglas formales, normas informales y mecanismos de cumplimiento) facilitan o dificultan la cooperación de los participantes y el nivel de eficiencia en el logro de resultados. En tal sentido, si bien todas las autoridades gubernamentales son importantes, en cuanto a la provisión de bienes públicos, las reglas, diseñadas por las comunidades involucradas, tenderán a tener en cuenta las características propias de sus diferentes realidades locales. De allí que, este enfoque multinivel defiende un espacio de acción propio para las jurisdicciones locales, donde el rol más importante del gobierno es ayudarlas a resolver sus conflictos de interés bajo los estándares sociales de justicia.

La definición de gobernanza territorial asumida en este artículo, valora al Estado, en tanto garante del bien común, como el actor que reconoce, regula e impulsa las acciones de otros actores, en una nueva manera de relacionar la actuación pública y su aplicación en los territorios, que haga realidad la participación ciudadana en la toma de decisiones, en la gestión y el control público.

A nivel práctico, se concuerda con las variables y sus indicadores seleccionados por Whittingham (2005), por cuanto provienen de un extenso estudio sobre proyectos en el área 
de gobernanza liderados por organismos internacionales ampliamente reconocidos, que han manejado el concepto en forma consistente y continuada, tales como, el Instituto de Gobernanza (The Institute on Governance - IOG), el Centro Canadiense para el Desarrollo Gerencial, el Banco Mundial, el Centro para el Diálogo Interamericano (Inter-American Dialogue) y, las Naciones Unidas, particularmente, el Centro para los Asentamientos Humanos (Hábitat) y el Programa de las Naciones Unidas para el Desarrollo (PNUD).

\section{Metodología}

La investigación, de alcance descriptivo, analiza las variables relacionadas con la gobernanza territorial, contenidas en el estudio de la SEnPlades: "Objetivos e indicadores de apoyo al Plan Nacional del Buen Vivir 2013- 2017", mediante la clasificación de Whittingham (2005); la cual contempla la definición de cuatro variables principales (Participación, Equidad, Rendición de cuentas, Eficiencia), siete variables secundarias o subvariables (Capacidad de res- puesta, Innovación administrativa y gerencial, Interacción Estado-ciudadanos-ONGs, Administración descentalizada, Creación de redes, Desarrollo del recurso humano) y, sus indicadores. No obstante, dado que la mayoría de las variables secundarias son en rigor componentes de las principales, se estimaron finalmente cinco variables (Tabla 1).

Por su parte, los indicadores fueron estudiados con base en los siguientes criterios:

- Análisis de la ficha metodológica de la SENPLADES

- Clasificación dentro de las variables consideradas

- Agrupación en tipos generales de indicadores dentro de cada variable, según la clasificación de Whittingham (2005)

- Desarrollo evolutivo en el nivel nacional

- Comparación en las zonas de planificación (Tabla 2).

Tabla 1.

\section{Definición de las variables de gobernanza territorial}

\begin{tabular}{|l|l|}
\multicolumn{1}{c|}{ Variable } & \multicolumn{1}{c}{ Definición } \\
\hline 1. Participación & $\begin{array}{l}\text { Posibilidad de cada persona de ser incluida en la toma de decisiones, en forma } \\
\text { directa o a través de representantes legítimos }\end{array}$ \\
\hline 2. Equidad & $\begin{array}{l}\text { Igualdad de oportunidades para el acceso a mejoras en el bienestar de la sociedad, } \\
\text { con énfasis en los miembros más vulnerables }\end{array}$ \\
\hline $\begin{array}{l}\text { 3. Rendición de } \\
\text { cuentas }\end{array}$ & $\begin{array}{l}\text { Responsabilidad de quienes gobiernan para con los gobernados, aceptando y } \\
\text { respetando los criterios de desempeño establecidos }\end{array}$ \\
\hline 4. Eficiencia & $\begin{array}{l}\text { Logro de resultados que se adecuan a las necesidades de la población, así como a la } \\
\text { maximización de los recursos }\end{array}$ \\
\hline $\begin{array}{l}\text { 5. Desarrollo del } \\
\text { recurso humano }\end{array}$ & $\begin{array}{l}\text { Realización sostenida de programas de reclutamiento, entrenamiento, desarrollo y } \\
\text { motivación de los servidores públicos }\end{array}$ \\
\hline
\end{tabular}

Fuente: Elaboración propia con base en Whittingham (2005). 
Tabla 2.

Zonas de Planificación del Ecuador

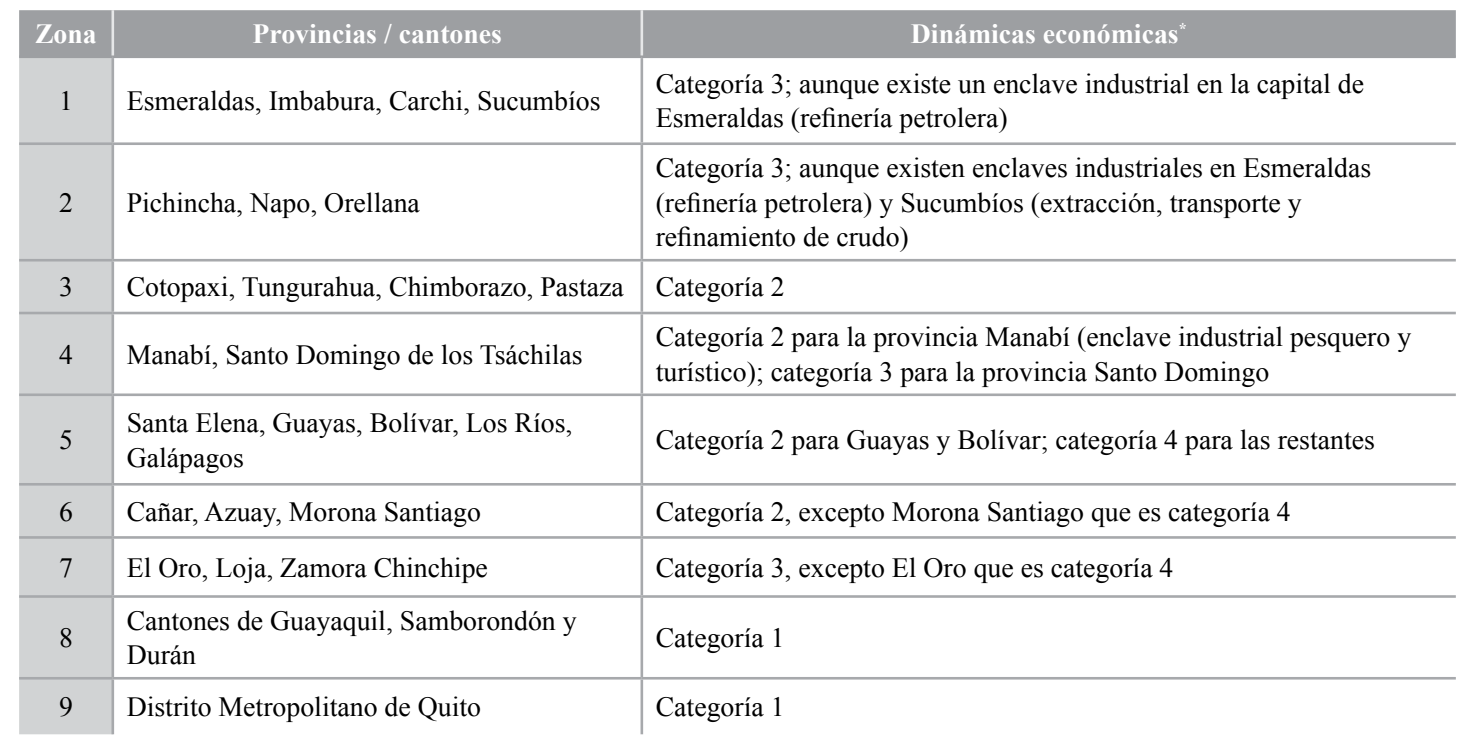

"Nota: En términos productivos se identifican cuatro espacios diferenciados en función de los índices de producción primaria, secundaria y terciaria: (1) Desarrollo industrial en crecimiento con servicios terciarios especializados

(2) Desarrollo primario consolidado con enclaves industriales básicos y/o turísticos

(3) Bajo desarrollo primario de mayor diversificación con puntales productivos

(4) Bajo desarrollo primario poco diversificado

Fuente: SENPLADES $(2012 ; 2017)$.

\section{Discusión de resultados}

En el documento de la Senplades se contemplan 149 indicadores, de los cuales 37 están directamente vinculados con la gobernanza territorial, según la clasificación de Whittingham (2005), representando el $25 \%$ del total ${ }^{1}$ (Tabla 3 ). El estudio incorpora estimaciones anuales hasta el año 2015, en su mayoría, con niveles de desagregación nacional, urbano/rural, y regiones de planificación, según el caso, y una disponibilidad de datos de entre cuatro a diez años de medición.

La variable Eficiencia presenta el mayor número de indicadores (65\%), seguida por Equidad (13.5\%), Participación (10.8\%), Rendición de cuentas (8\%) y Desarrollo del recurso humano $(2.7 \%)$, a partir de lo cual se pueden resaltar tres aspectos. Primero, poco fortalecimiento institucional con base en la capacitación del servidor público y el cambio de pautas culturales. Segundo, subestimación de procesos de rendición de cuentas a los que delegaron el poder y de representación de grupos de interés en las decisiones que los afectan, evidenciando un modelo de gobernanza centrado en el Estado, a contrapelo de los dispositivos constitucionales y legales. Tercero, desatención de los mecanismos institucionales de participación ciudadana, sugiriendo la práctica de un ritual vacío de participación en las diferentes instancias del poder (Arnstein, 1969); así como el descenso en los niveles de asociatividad público-privada, la cooperación en proyectos sociales y la incursión en campos novedosos de economía territorial, esenciales para el desarrollo local.

Para la variable Eficiencia destaca la ausencia de indicadores sobre: innovaciones administrativas/gerenciales de los gobiernos locales; administración descentralizada; y, creación de redes de cooperación con otros gobiernos y entidades. Todos los cuales son evidencias de buena gobernanza, en razón de potenciar la modernización, la vinculación de las comunidades y la asociatividad entre municipios, para el desarrollo territorial. 
Tabla 3.

Clasificación de los Indicadores en las variables de gobernanza territorial

\begin{tabular}{|c|c|c|c|c|c|}
\hline $\begin{array}{l}\text { Variable de } \\
\text { Gobernanza }\end{array}$ & Tipos de Indicadores & $\mathbf{N}^{\circ}$ & $\mathbf{N}^{\circ}$ total & $\%$ & Fuente de datos \\
\hline \multirow{4}{*}{ Participación } & $\begin{array}{c}\text { Acciones en ejercicio de } \\
\text { derechos }\end{array}$ & 01 & 04 & 10.8 & $\begin{array}{l}\text { - INEC / Encuesta Nacional de Empleo, } \\
\text { Desempleo y Subempleo (ENEMDU) } \\
\text { - Agencia de Regulación y Control de } \\
\text { Telecomunicaciones }\end{array}$ \\
\hline & $\begin{array}{c}\text { Participación en actividades } \\
\text { políticas }\end{array}$ & 01 & & & \\
\hline & $\begin{array}{l}\text { Participación en trabajo } \\
\text { comunitario }\end{array}$ & 01 & & & \\
\hline & $\begin{array}{l}\text { Participación de medios } \\
\text { comunitarios }\end{array}$ & 01 & & & \\
\hline \multirow{3}{*}{ Equidad } & $\begin{array}{l}\text { Acceso a servicios básicos de } \\
\text { sectores en desventaja }\end{array}$ & 01 & 05 & 13.5 & $\begin{array}{l}\text { - INEC / ENEMDU, con base en modelos } \\
\text { econométricos }\end{array}$ \\
\hline & Representación de las mujeres & 02 & & & \\
\hline & Cifras de desempleo & 02 & & & \\
\hline \multirow{2}{*}{$\begin{array}{l}\text { Rendición de } \\
\text { cuentas }\end{array}$} & $\begin{array}{l}\text { Cumplimiento de metas de los } \\
\qquad \text { GAD }^{*}\end{array}$ & 01 & 03 & 8.0 & $\begin{array}{l}\text { - Ministerio de Finanzas } \\
\text { - SENPLADES }\end{array}$ \\
\hline & $\begin{array}{l}\text { Grado de delegación de } \\
\text { autoridad y transferencia } \\
\text { desde el gobierno central }\end{array}$ & 02 & & & \\
\hline \multirow{4}{*}{ Eficiencia } & $\begin{array}{l}\text { Reducción de la población } \\
\text { bajo línea de pobreza }\end{array}$ & 03 & 24 & 65.0 & $\begin{array}{l}\text { - INEC / ENEMDU } \\
\text { - Latinobarómetro }\end{array}$ \\
\hline & $\begin{array}{c}\text { Reducción de índices de } \\
\text { trabajo infantil }\end{array}$ & 02 & & & \\
\hline & $\begin{array}{c}\text { Aumento de participación en } \\
\text { áreas de bienestar (vivienda, } \\
\text { educación) }\end{array}$ & 13 & & & \\
\hline & $\begin{array}{c}\text { Grado de satisfacción de la } \\
\text { población }\end{array}$ & 06 & & & \\
\hline $\begin{array}{l}\text { Desarrollo del } \\
\text { recurso humano }\end{array}$ & $\begin{array}{l}\text { Programas de capacitación } \\
\text { para servidores públicos }\end{array}$ & 01 & 01 & 2.7 & - INEC / ENEMDU \\
\hline Total & & & 37 & 100 & \\
\hline
\end{tabular}

*Gobiernos Autónomos Descentralizados.

Fuente: Elaboración propia con base en SenPlades (2016).

Para la variable Equidad no hay indicadores sobre la representación política de las mujeres, inclusión de grupos excluidos en procesos consultivos, ni destinación de recursos a poblaciones vulnerables, de los que los últimos son particularmente importantes en un país multicultural y plurinacional como el Ecuador. La Rendición de cuentas es poco estimada por la SENPLADES, ignorando indicadores que valoren el grado de atención a los reclamos de los ciudadanos, capacidad de respuesta de los servidores públicos, existencia de mecanismos para conocer las preferencias/necesidades de la gente, de procedimientos de evaluación de metas, así como de mecanismos de transparencia de la gestión pública.

En cuanto a la Participación, no hay indicadores sobre asociatividad público-privada, interacción Estado-ciudadanos-ONGs o involucramiento de la sociedad civil en la cosa pública, lo cual es llamativo considerando la extensa labor que históricamente realizan ONGs nacionales, en los ámbitos de reducción de la pobreza, medio ambiente, observación electoral, transparencia, desarrollo local, enfoque de 
género, entre otros (Chiriboga, 2014). En la variable Desarrollo del recurso humano, no hay indicadores específicos sobre la existencia y cubrimiento de programas sostenidos de reclutamiento y selección basados en el mérito y las capacidades, ni de sistemas de clasificación y compensación.

\section{Impacto territorial de las políticas públicas}

Para propósitos de comparación de las zonas de planificación, se efectúa un análisis longitudinal y, para algunos indicadores, se muestran los datos del año 2015, por ser el último año del estudio.

\section{Variable Participación}

Las "acciones de participación" y la "participación en actividades políticas", tienen comportamientos evolutivos fluctuantes con moderada tendencia a subir en todas las zonas, siendo la zona 2 la más activa. La "participación ciudadana en trabajo comunitario" tuvo un gran incremento en el año 2009 pero descendió en el 2015, mientras la "participación de medios comunitarios" es ínfima con respecto al total de frecuencias. Tales aspectos requieren ser fortalecidos, por cuanto representan formas complementarias que permiten generar nuevas maneras y escenarios para la construcción de una ciudadanía más activa y participativa en la gestión de políticas públicas (Ramos, 2015). La Figura 1 muestra el comportamiento de los dos primeros indicadores para el 2015, destacando sobre todo la zona 2 (Pichincha, Napo, Orellana).

\section{Variable equidad}

En cuanto al "acceso a servicios básicos de sectores en desventaja" el único indicador (cobertura de servicio eléctrico en áreas rurales), experimentó avances en todas las zonas, desde un valor nacional de $88,66 \%$ (año 2006) hasta 95,10\% (año 2015). Los dos indicadores de "representación de las mujeres" tuvieron comportamientos diferentes, por una parte, hubo un gran incremento del "porcentaje de amas de casa con seguridad social contributiva" en todas las zonas, pero la "brecha del ingreso laboral entre mujeres y hombres" se mantuvo casi constante, indicando que no ha habido un mejoramiento sustancial en esa área. En cuanto a los indicadores de "desempleo", se encuentran mejoras en la "tasa de ocupación digna", en todas las zonas de planificación, mientras la "tasa de desempleo" tiende a la baja.

En la Figura 2 se observa que, para el año 2015 , la brecha del ingreso laboral entre mujeres y hombres es bastante similar en todas las zonas excepto la 8 (cantones de Guayaquil, Samborondón y Durán), una de las de mayor dinamismo económico, mientras que la tasa de

Figura 1.

Indicadores de participación, año 2015
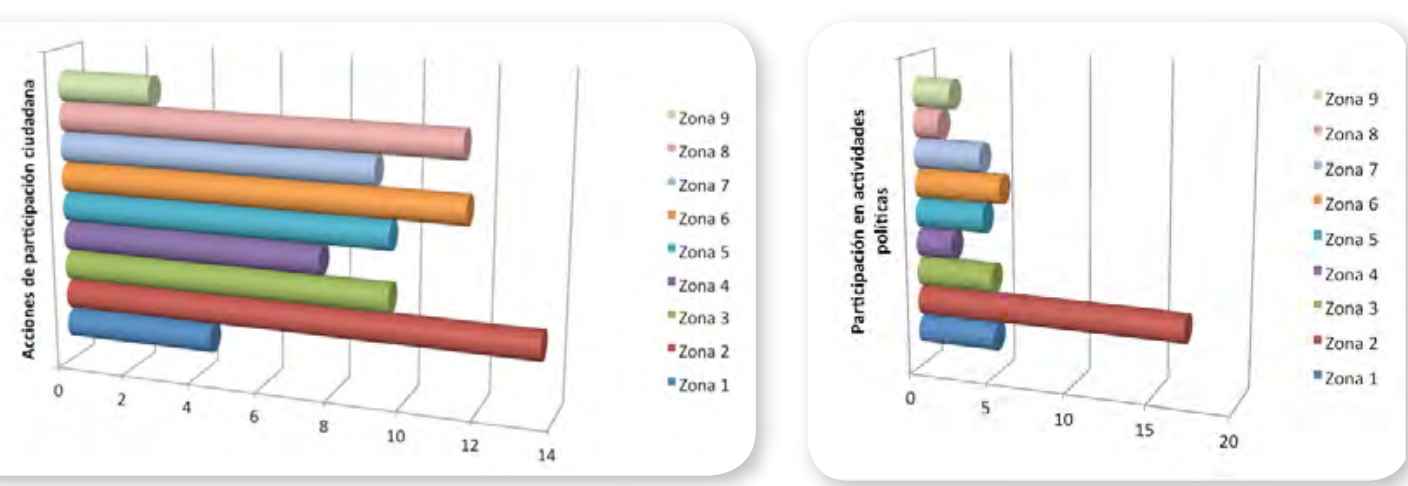

Fuente: Elaboración propia con base en SENPLADES (2015). 
Figura 2.

Indicadores de equidad, año 2015

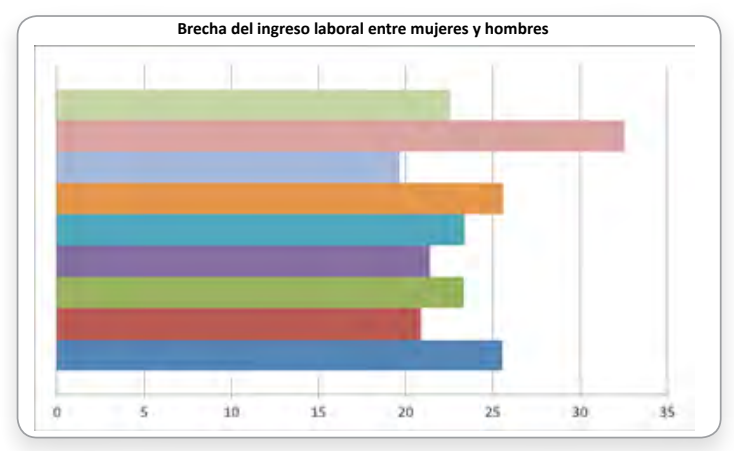

Fuente: Elaboración propia con base en SEnPLades (2015).

desempleo es menor para las zonas 3 (Cotopaxi, Tungurahua, Chimborazo, Pastaza) y 6 (Cañar, Azuay, Morona Santiago), las cuales representan territorios de desarrollo primario consolidado con enclaves industriales básicos y/o turísticos.

\section{Variable rendición de cuentas}

La Figura 3 muestra como el único indicador para "cumplimiento de metas de los GAD" (índice de cumplimiento de metas promedio de los GAD), ha experimentado preocupantes bajas sucesivas durante el período de medición; mientras que los dos indicado-

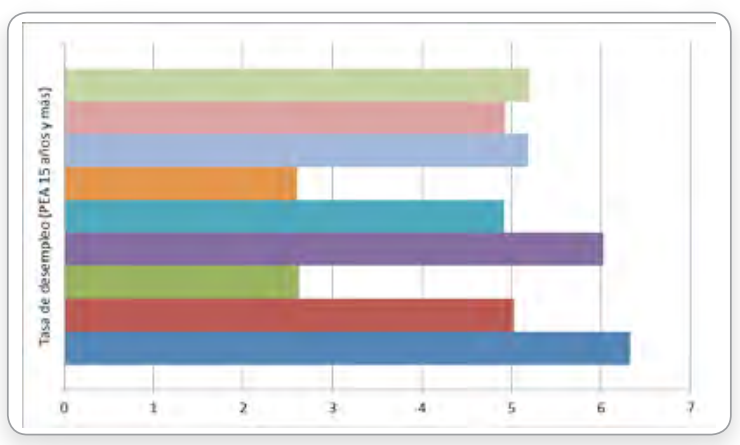

res sobre "grado de delegación de autoridad y transferencia desde el gobierno central" (coeficiente de Gini de transferencia de recursos a los GAD y coeficiente de Gini de transferencia per cápita del Gobierno Central a los GAD), experimentan una trayectoria descendente, implicando una mejora en la distribución de los recursos a las provincias, cantones y parroquias.

\section{Variable eficiencia}

Acá se encuentra la mayoría de los indicadores, mostrando diferentes grados de éxito de las políticas públicas correspondientes.

Figura 3.

Indicadores de rendición de cuentas. Análisis longitudinal

Coeficiente de Gini para la transferencia de recursos a los GAD

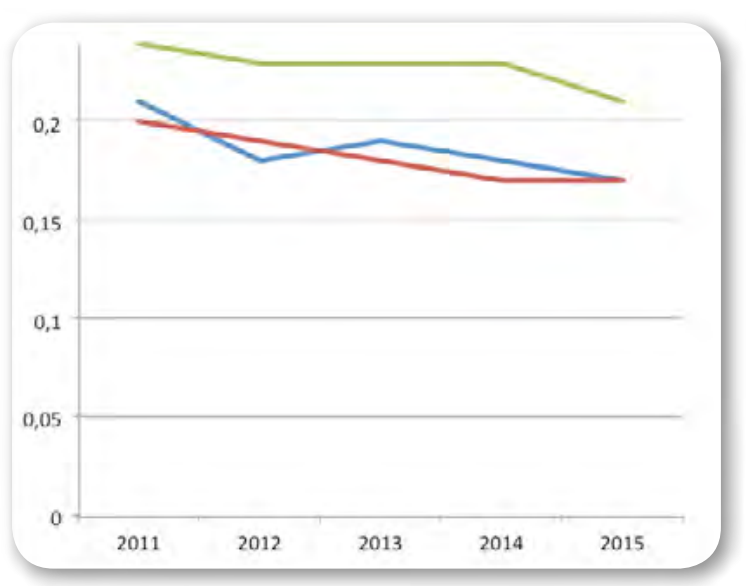

Fuente: Elaboración propia con base en SenPLADEs (2015).
Índice de cumplimiento de metas promedio de los GAD

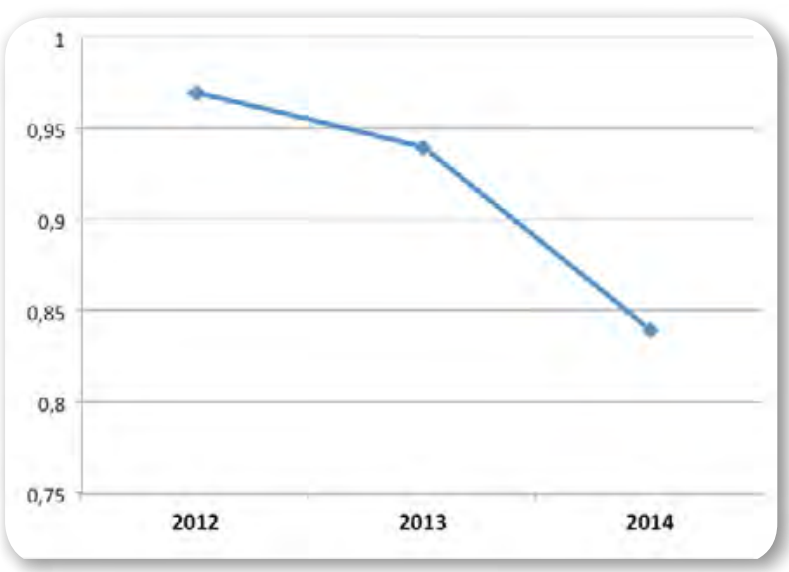


Los cinco índices relativos a "reducción de la población bajo la línea de pobreza" y a la "reducción de índices de trabajo infantil" presentan una tendencia sostenida descendente en todas las zonas de planificación. Entre los 13 indicadores relativos al "aumento de participación en áreas de bienestar (vivienda, educación)", destaca el déficit cualitativo de la vivienda que mantuvo una trayectoria temporal casi invariable en todas las zonas, excepto en la 9 (Distrito Metropolitano de Quito), donde muestra importantes reducciones. Igualmente, el porcentaje de hogares que habitan en viviendas propias casi no ha cambiado durante diez años, implicando que no ha habido un impulso público decisivo y/o que las políticas no han tenido impacto.

En el ámbito educativo se encuentran ocho índices con trayectorias temporales fluctuantes. Hay mejorías sustanciales en las tasas de analfabetismo, analfabetismo funcional, analfabetismo digital, población con primaria completa (86\% nacional año 2015$)$ y con secundaria completa (45\% nacional año 2015), pero existe estancamiento en educación superior tanto en el acceso (18\% nacional año $2015)$ como en la matrícula ( $21 \%$ nacional año
2015). Esto es preocupante, pues el área de la educación superior tiene gran relevancia para la formación de recursos humanos en actividades científicas e intelectuales, así como en procesos de generación y transferencia de tecnologías, representando uno de los indicadores territoriales de innovación y conocimiento (Caravaca, García y González, 2012).

En cuanto a los seis indicadores del "grado de satisfacción de la población" (escala $0-10)$, no hay diferencias marcadas, ni temporales ni regionales, para la educación $(6,78$ nacional, año 2015), salud (5,84 nacional año 2015), subvenciones y ayudas (7,41 nacional, año 2015), transporte público $(6,02$ nacional, año 2015). En contraste, existe un notable descenso en la percepción sobre las instituciones públicas (de 33,29\% nacional año 2011 a 27,59\% nacional año 2015), siendo las más bajas en las zonas 1 (Esmeraldas, Imbabura, Carchi, Sucumbíos) y 9 (Distrito Metropolitano de Quito); mientras que la satisfacción con la democracia se ha incrementado desde el año 2006 (23\%) hasta el 2015 (59,63\%). Todo ello evidencia una percepción general positiva hacia el desempeño oficial.

Figura 4.

Indicadores de eficiencia, año 2015
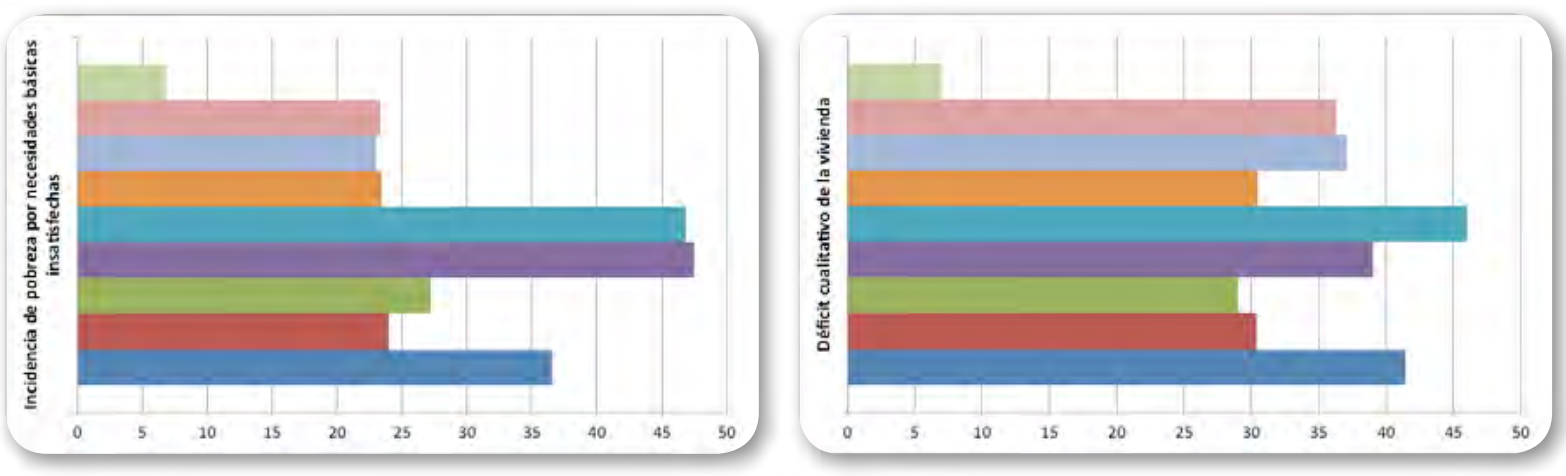

Fuente: Elaboración propia con base en SenPLADES (2015). 


\section{Variable desarrollo del recurso humano}

Del total de 149 indicadores del informe de la Senplades, hay un solo indicador sobre "programas de capacitación para servidores públicos" (capacitación de los servidores públicos), en conocimientos y destrezas que propendan a su desarrollo profesional, técnico y personal, el cual muestra un comportamiento ascendente desde el año 2006 (48,38\% nacional) hasta el 2012 (60\% nacional), a partir del cual inicia un descenso sostenido alcanzando $51,64 \%$ para el año 2015. La Figura 5 muestra que los mayores niveles de capacitación se encuentran en las zonas 9 (Distrito Metropolitano de Quito), 3 (Cotopaxi, Tungurahua, Chimborazo, Pastaza) y 1 (Esmeraldas, Imbabura, Carchi, Sucumbíos).

Figura 5. Indicadores de desarrollo del recurso humano, año 2015

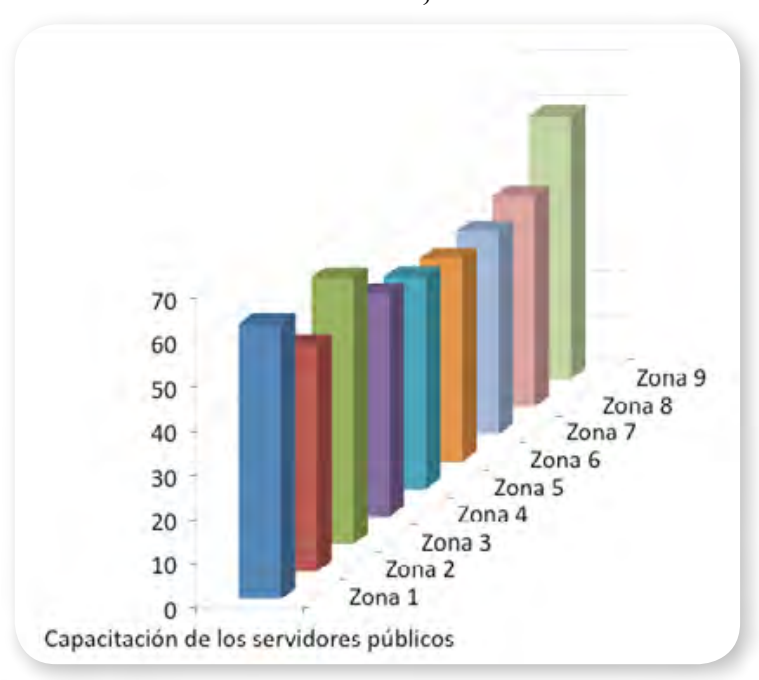

Fuente: Elaboración propia con base en SENPLAdES (2015)

\section{Consideraciones finales}

De acuerdo con la clasificación utilizada en esta investigación, existe ausencia de indicadores básicos en todas las variables consideradas por la Senplades en su análisis de cumplimiento de los objetivos del Plan Nacional del Buen Vivir 2013- 2017, por lo que se carece de información sobre importantes atributos de la dinámica territorial del desarrollo en las zonas de planificación del Ecuador, especialmente la coordinación de las políticas desde distintos niveles institucionales y a diferentes escalas espaciales, la articulación y concertación de actores, la conformación de redes, entre otros elementos esenciales de la gobernanza territorial.

Aunque las zonas de planificación del Ecuador pueden distribuirse en cuatro categorías económicas, la mayor superficie del territorio nacional se caracteriza como de "bajo desarrollo primario de mayor diversificación con puntales productivos", identificándose la primacía del sector primario con puntales productivos como la palma africana, los cultivos de cacao y de maíz, el cultivo de papa, la producción de textiles y productos de cuero, y la extracción de petróleo. Dadas estas circunstancias son requeridas, junto a las políticas nacionales, la implementación de específicas políticas regionales y sectoriales que impulsen el desarrollo, con base en la habilidad de los gobiernos locales para forjar relaciones de cooperación con los actores sociales del territorio, con otras entidades y gobiernos, así como en la capacidad de introducir innovaciones en sus procedimientos administrativos y en la participación de las comunidades.

Consecuentemente, resulta preocupante evidenciar carencias o poco fortalecimiento institucional en aspectos tales como, la capacitación del servidor público, los procesos de rendición de cuentas y de representación de grupos de interés, y los mecanismos de participación ciudadana; aunque en líneas generales se aprecien mejorías a nivel regional en la mayoría de los indicadores, como impacto positivo de las políticas implementadas. 


\section{Referencias}

Arnstein, S. (1969). A ladder of citizen participation. Journal of the American Planning Association, 35(4), 216-224.

Arocena, J. (2002). El desarrollo local. Un desafio contemporáneo. Uruguay: TaurusUniversidad Católica.

Arocena, J. (2011). Descentralización: condiciones, riesgos y desafíos. En J. Fernández, y M. Trocello (Coords.). Intermunicipalidad y desarrollo local: hacia una teoría común iberoamericana de la intermunicipalidad (pp. 25-46). Madrid: Unión Iberoamericana de Municipalistas.

Boisier, S. (2004). Desarrollo territorial y descentralización. El desarrollo en el lugar y en las manos de la gente. Revista Eure, 30(90), 27-40.

Boisier, S. (2011). El territorio en la contemporaneidad (la recuperación de las políticas territoriales). Revista Líder, 18(13), 9-24.

Canto, R. (2012). Gobernanza y democracia. De vuelta al río turbio de la política. Gestión y Política Pública, 21(2), 333-374.

Caravaca, I., García, A., y González, G. (2012). Hacia la integración de Andalucía en la sociedad del conocimiento: algunas estrategias públicas regionales, metropolitanas y locales. Ciudad y Territorio: Estudios territoriales, 49(172), 263-280.

Cerrillo i Martínez, A. (2005). La gobernanza hoy: 10 textos de referencia. Madrid: Instituto Nacional de Administración Pública.

Comisión Europea. (2008). Un enfoque de la UE hacia la gobernanza local democrática, la descentralización y el desarrollo territorial. Recuperado de: http://www.cities-localgovernments.org/ uclg/upload/news/newsdocs/ec_issues_ paper_es.pdf
Constitución Política del Ecuador. (2008). Asamblea Constituyente. Recuperado de: http://www.produccion.gob.ec/wpcontent/uploads/downloads/2012/07/ Normas_Constitucionales.pdf

Gallicchio, E. (2010). El desarrollo local: ¿territorializar políticas o generar políticas territoriales? Reflexiones desde la práctica. Eutopia, (1), 11-23.

Guttman, D. (2004). De gobierno a gobernanza: La nueva ideología de la rendición de las cuentas, sus conflictos, sus defectos y sus características. Gestión y Política Pública, 13(1), 5-40.

Jorquera, D. (2011). Gobernanza para el Desarrollo Local. [Documento de Trabajo $\mathrm{N}^{\circ}$ 6. Proyecto Conocimiento y Cambio en Pobreza Rural y Desarrollo]. Santiago: Rimisp. Recuperado de: https://www.rimisp.org/wp-content/files mf/1366307608n952011gobernanzapara desarrollolocaljorquera.pdf

Klijn, E. H. (1998). Redes de políticas públicas: Una visión general. En W. J. Kickert, y J. Koppenjan, (Coord.). Managing Complex Networks. London: Sage.

Natera, A. (2004). La noción de gobernanza como gestión pública participativa y reticular. Documentos de Trabajo "Política y Gestión" Vol. 2 Madrid: Universidad Carlos III de Madrid.

Ostrom, E. (2005). Understanding institutional diversity. Princeton: Princeton University Press.

Ostrom, E. (2009). A polycentric approach for coping with climate change. World Bank Policy Research Working Paper Series, 5095. Washington D.C: World Bank.

Ostrom, E. (2010). Beyond markets and states: Polycentric governance of complex economic systems, American Economic Review, 100(3), 641-672. 
O’Toole, L. (2005). Las implicaciones para la democracia de una burocracia en red. En A. Cerrillo i Martínez, (Coord.). La gobernanza hoy: 10 textos de referencia (pp. 245-262). Madrid: Instituto Nacional de Administración Pública.

Peters, B. G. (2007). Globalización, gobernanza y Estado: algunas proposiciones acerca del proceso de gobernar. Revista del CLAD Reforma y Democracia, (39), 33-50.

Peters, B. G. y Pierre, J. (2005). ¿Por qué ahora el interés por la gobernanza?. En A. Cerrillo i Martínez, (Coord.). La gobernanza hoy: 10 textos de referencia (pp. 37-56). Madrid: Instituto Nacional de Administración Pública.

Ramos, H. (2015). Información y ciudadanía, una propuesta desde la gobernanza. Investigación Bibliotecológica, 29(67), 113-140.

Revesz, B. (2005). Gobernabilidad democrática, descentralización y desarrollo territorial local y regional. Nueva ruralidad y competitividad territorial. Lima: Centro Ideas.

Rhodes, R. A. W. (2005). La nueva gobernanza: gobernar sin gobierno. En: A. Cerrillo i Martínez, (Coord.). La gobernanza hoy: 10 textos de referencia (pp. 99-122), Madrid: Instituto Nacional de Administración Pública.

Secretaría Nacional de Planificación y Desarrollo [Senplades] (2017). Plan Nacional de Desarrollo 2017-2021. Toda una vida. Recuperado de:

http://www.planificacion.gob.ec/wpcontent/uploads/downloads/2017/10/ PNBV-26-OCT-FINAL_0K. compressed1.pdf

Secretaría Nacional de Planificación y Desarrollo [SENPLADEs] (2016). Objetivos e indicadores de apoyo al Plan Nacional del Buen Vivir 2013-2017. Recuperado de: http://indestadistica.sni.gob. ec/QvAJAXZfc/opendoc.
htm?document=SNI.qvw\&host=QVS@

kukuri\&anonymous=truehttp://

indestadistica.sni.gob.ec/QvAJAXZfc/

opendoc.htm?document=SNI.

qvw\&host=QVS@kukuri\&anony

mous $=$ true \&bookmark=Document/

BM82\&select $=$ LB729,Acciones, $\% 20$

de, $\% 20$ participaci $\%$ C3\%B3n, $\% 20$

ciudadana.

Consultado el 01/11/16

Secretaría Nacional de Planificación y Desarrollo - [Senplades] (2013). Plan Nacional para el Buen Vivir 2013-2017. Quito. Recuperado de:

http://documentos.senplades.gob.

ec/Plan $\% 20$ Nacional $\% 20$ Buen $\% 20$

Vivir\%202013-2017.pdf

Consultado el 01/08/16.

Secretaría Nacional de Planificación y Desarro110 - [Senplades] (2012). Proceso de desconcentración del Ejecutivo en los niveles administrativos de planificación. Folleto Informativo I. Quito. Recuperado de: http://www.planificacion. gob.ec/wp-content/uploads/ downloads/2012/10/Folleto_informativoDesconcentracion2012.pdf.

Torres, V. (2016). Desarrollo local y municipios en Ecuador. Avances y retrocesos. IV Congreso Internacional de Desarrollo Local, (9-12 de noviembre de 2016), Lima.

Valarezo, G. (2004). El Estado y la cuestión local en el Ecuador contemporáneo. En G. Valarezo y V. Torres, (Coords.). El desarrollo local en el Ecuador: Historia, actores y métodos (pp. 77-117). Quito: Abya Yala.

Verdesoto, L., y Ardaya, G. (2011). Continuidad y cambios en el desarrollo local y regional. Una aproximación al caso ecuatoriano. Polémica, 6(1). Recuperado de: http://revistas.usfq.edu.ec/index.php/ polemika/article/view/391 
Vigil, J., y Ockstat, W. (2015). Las deudas pendientes de la gobernanza del desarrollo regional en Argentina. Pampa (Santa $\mathrm{Fe}$ ), (12), 187-215.

Whittingham, M. (2005). Aportes de la teoría y la praxis para la nueva gobernanza. $R e$ vista del CLAD Reforma y Democracia, (33), 1-15.

\section{Notas}

1 En este estudio no fueron considerados algunos índices específicos de educación y salud, que si bien pueden medir el grado de Eficiencia de las políticas públicas, representan subcomponentes o desagregaciones de indicadores más generales. 
